\title{
Design and Validation of a Virtual Player for Studying Interpersonal Coordination in the Mirror Game
}

\author{
Chao Zhai, Francesco Alderisio, Piotr Słowiński, Krasimira Tsaneva-Atanasova and \\ Mario di Bernardo, Fellow, IEEE
}

\begin{abstract}
The mirror game has been recently proposed as a simple, yet powerful paradigm for studying interpersonal interactions. It has been suggested that a virtual partner able to play the game with human subjects can be an effective tool to affect the underlying neural processes needed to establish the necessary connections between the players, and also to provide new clinical interventions for rehabilitation of patients suffering from social disorders. Inspired by the motor processes of the central nervous system (CNS) and the musculoskeletal system in the human body, in this paper we develop a novel interactive cognitive architecture based on nonlinear control theory to drive a virtual player (VP) to play the mirror game with a human player (HP) in different configurations. Specifically, we consider two cases: the former where the VP acts as leader and the latter where it acts as follower. The crucial problem is to design a feedback control architecture capable of imitating and following or leading a human player in a joint action task. Movement of the end-effector of the VP is modeled by means of a feedback controlled Haken-Kelso-Bunz (HKB) oscillator, which is coupled with the observed motion of the HP measured in real time. To this aim, two types of control algorithms (adaptive control and optimal control) are used and implemented on the HKB model so that the VP can generate a human-like motion while satisfying certain kinematic constraints. A proof of convergence of the control algorithms is presented in the paper together with an extensive numerical and experimental validation of their effectiveness. A comparison with other existing designs is also discussed, showing the flexibility and the advantages of our control-based approach.
\end{abstract}

Index Terms-Motor coordination, virtual player, mirror game, joint action, nonlinear control.

\section{INTRODUCTION}

$\mathbf{T}$ HE emergence of coordinated behavior between humans is a common phenomenon in many areas of human endeavor. Examples include improvisation theater, group dance, music playing, team sports and parade marching [1]. At the core of the interaction between the players lies a fundamental

Chao Zhai, Francesco Alderisio and Mario di Bernardo are with Department of Engineering Mathematics, University of Bristol, Merchant Venturers' Building, Woodland Road, Clifton, BS8 1UB, United Kingdom. e-mail: chao.zhai@bristol.ac.uk, f.alderisio@bristol.ac.uk, m.dibernardo@bristol.ac.uk.

Piotr Słowiński and Krasimira Tsaneva-Atanasova are with College of Engineering, Mathematics and Physical Sciences, University of Exeter, United Kingdom. e-mail: slowinski.piotr@gmail.com, k.tsanevaatanasova@exeter.ac.uk

Mario di Bernardo is also with Department of Electrical Engineering and Information Technology, University of Naples Federico II, 80125 Naples, Italy.

Manuscript received August 26, 2015; revised July 25, 2016. feedback mechanism where each player adapts her/his motion in response to the observed movement of the other.

To study this intriguing phenomenon, the mirror game has been recently proposed as a simple, yet effective paradigm. In its simplest formulation, the mirror game features two people imitating each other's movements at high temporal and spatial resolution [2]. The game can be played in different experimental conditions: the former where one of the players leads and the other has to follow the leader's motion (LeaderFollower condition); the latter where the two players create joint synchronized movement (Joint Improvisation condition).

The theory of similarity in social psychology suggests that people prefer to team up with others possessing similar morphological and behavioral features, and that interpersonal coordination is enhanced if their movement shares similar kinematic features [3], [4]. Further evidence suggests that motor processes caused by interpersonal coordination are strictly related to mental connectedness. Specifically, motor coordination between two people contributes to social attachment [5].

As suggested in [5], coordination games can therefore be used to help people suffering from social disorders improve their social skills. Also they can be effectively exploited in social robotics to enhance attachment, coordination and rehabilitation during human-robot interactions [6]. For this reason, it has been proposed that creating a VP or avatar able to coordinate its motion with that of a HP can be extremely useful to study the onset of coordination and how it is affected by similarity/dissimilarity between the players' motion characteristics [7]. A VP can also be used for diagnostics and rehabilitation of patients suffering from social disorders as recently proposed in [8].

The aim of this paper is the design of a novel interactive cognitive architecture (ICA), based on nonlinear control theory, able to drive a VP to play the mirror game with a human either as a leader or as a follower. Contrary to previous approaches in the literature, the proposed ICA allows for bi-directional feedback between the HP and the VP, which was shown to be one of the key components in interpersonal coordination [9], while guaranteeing that the motion of the VP, when interacting in real-time with the HP, exhibits certain desired kinematic features. When playing as a follower, the ICA needs to guarantee that, while exhibiting the desired movement properties, the VP tracks as closely as possible the motion of the human leader. When playing as the leader, the 
ICA needs instead to generate new interesting motion. The proposed ICA can also be integrated into a humanoid robot to achieve the desired dual-arm coordination [10].

In this paper we take the view that the design of such an architecture is fundamentally a nonlinear control design problem where, given some reference input, the architecture has to drive the VP onto a desired motion which is a function of the movement of the human player being sensed during the game. We explore two different approaches, one based on adaptive control, the other on optimal control. Our control architecture mimics the two fundamental actions which have been suggested to be at the core of the emergence of motor coordination between two or more effectors in biological systems: feedback and feedforward [11], [12], [13]. Specifically, the motor system is able to correct the deviation from the desired movement with the aid of feedback control, whilst feedforward control allows it to reconcile the interdependency of the involved effectors and preplan the response to the sensory incoming information [12], [13], [14].

We show experimentally that the proposed control architectures are able to effectively drive the VP to play the mirror game while generating motion with desired kinematic properties. In particular, we use the concept of Individual Motor Signature (IMS) recently proposed in [15], [16] to characterize the motion of an individual player and evaluate how similar/dissimilar the motion of two different individuals is. Following our approach we are able to show that the VP driven by the cognitive architecture presented in the rest of this paper can play the mirror game either as a leader or a follower while exhibiting a desired IMS.

Relevant previous work in the literature includes the generation of human-like movements [17], the development of a mathematical model to explain the coordination dynamics observed experimentally in the mirror game [2], and the human dynamic clamp paradigm proposed in [7], [18], [19] where the use of a virtual partner driven by appropriate mathematical models is proposed to study human motor coordination. These previous approaches will be used to investigate and compare the performance of the novel strategy presented in this paper. We wish to emphasize that the control algorithms developed and validated in what follows can be also effectively used for trajectory planning to enhance human-robot coordination in joint interactive tasks.

The rest of the paper is organized as follows. Mirror game set-up, problem statement and motor signature are discussed in Section II before presenting the schematic of the proposed cognitive architecture in Section IIII The feedback control strategies at the core of the ICA are developed and analyzed in Section $[\mathrm{IV}$ and $\mathrm{V}$. The experimental validation of the control algorithms is presented in Section VI where experimental results are discussed showing the effectiveness of the proposed strategies. A comparison with other existing approaches is also carried out. Finally, conclusions and suggestions for future work are drawn in Section VII.

\section{The Mirror Game Problem}

Investigation of interpersonal coordination requires appropriate experimental paradigms. A typical paradigm recently

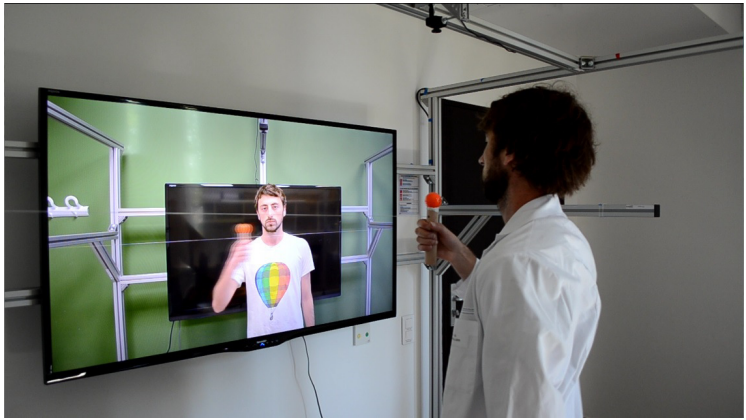

Fig. 1. Experimental set-up of the mirror game between a VP and a HP at the University of Montpellier, France (see [8] for further details).

proposed in the literature is the mirror game, which involves two people imitating each other's movements at high temporal and spatial resolution [2]. It can be played in two different conditions: Leader-Follower condition, where the follower attempts at tracking the leader's motion as accurately as possible, and Joint Improvisation condition, where the players jointly coordinate and synchronize their movements without any of the two being designated as leader or follower.

Our set-up is inspired by that in [2]. Specifically, a small orange ball is mounted onto a string, which the HP can move back and forth along the string itself. In the meanwhile, the VP on the opposite screen moves its own ball on a parallel string with the same length (see Fig. 11. In this implementation of the mirror game, two players (a HP and a VP) are required to move their respective ball back and forth and synchronize their movement. Here, we assume that the game is played in a Leader-Follower condition.

The position of the ball moved by the HP is detected by a camera. A feedback control strategy then needs to be designed in order to generate the trajectory of the ball moved by the VP. Such a trajectory can then be provided to the on-board controllers of the VP (robot or computer avatar) as the desired trajectory for its end effector.

To solve this control problem so that the VP motion presents similar features to the motion of a human player, it is necessary to choose an appropriate model of the VP motion that can then be controlled using a nonlinear feedback strategy. To this purpose, here we use the Haken-Kelso-Bunz oscillator, which was first proposed in [20] as a model able to capture the observations made in experiments on human bimanual coordination. The model consists of two nonlinearly coupled nonlinear oscillators described by

$$
\ddot{z}+\left(\alpha \dot{z}^{2}+\beta z^{2}-\gamma\right) \dot{z}+\omega^{2} z=\left[a+b(z-w)^{2}\right](\dot{z}-\dot{w})
$$

where $z, \dot{z}$ represent position and velocity of finger 1 , and $w, \dot{w}$ those of finger 2, modeled by a replica of (1) obtained by swapping $w, \dot{w}$ with $z, \dot{z} ; a$ and $b$ are the coupling parameters, and $\alpha, \beta, \gamma$ and $\omega$ characterize the response of each uncoupled finger when subject to some reference signal. It is worthy of note that, other than describing intrapersonal motor coordination, the HKB oscillator was suggested in the literature as a paradigmatic example of human motor coordination even beyond the simple bimanual experiments 

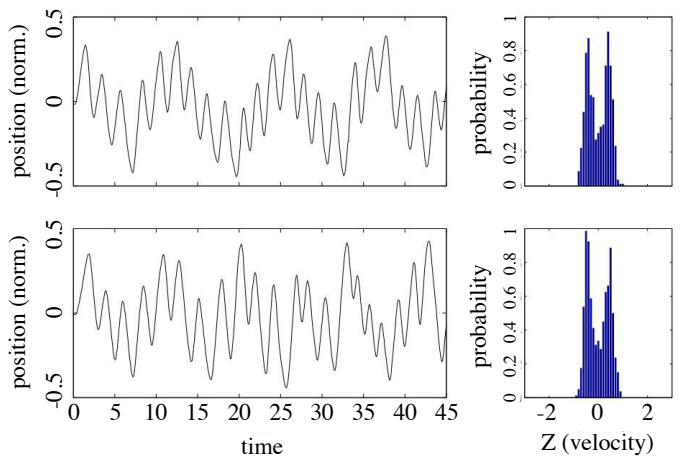

(a) Same player
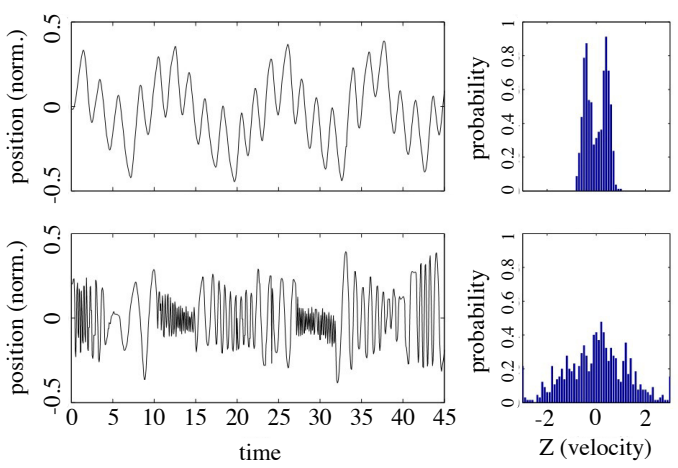

(b) Two different players

Fig. 2. Position time series and corresponding PDF of velocity time series for the same player (a) and two different players (b).

it was originally introduced for [7], [18], [20], [21]. Indeed, the HKB model was used to describe interpersonal motor coordination involving two different people [22], [23], [24] or a group of three or more persons [25], and it was shown to be better qualified as end effector model in the mirror game than other systems [26].

Solving the mirror game can be formulated as the following control problem. Given a nonlinear HKB oscillator of the form

$$
\left\{\begin{array}{l}
\dot{x}(t)=y(t) \\
\dot{y}(t)=-\left(\alpha y^{2}(t)+\beta x^{2}(t)-\gamma\right) y(t)-\omega^{2} x(t)+u(t)
\end{array}\right.
$$

where $x$ and $\dot{x}$ refer to the position and velocity of the end effector of the VP, respectively, and $u$ is an external control input, the problem is to design a feedback controller $u$ such that $x$ achieves bounded asymptotic tracking of the position of the HP, while expressing some desired kinematic features.

As metrics to characterize the kinematic properties of the motion of an individual playing the game we use the concept of individual motor signature (IMS), recently introduced in [15], [16]. It has been shown that the IMS is time invariant and unique for each player. It is defined in terms of the velocity profile (or distribution) of the player's motion in solo trials. To quantify how similar or dissimilar the signatures of two different players are, we use the earth mover's distance (EMD) between any two probability distribution functions (PDF) of their velocity time series [16], [27]. The EMD between two PDFs $p_{1}$ and $p_{2}$ can be computed as $\operatorname{EMD}\left(p_{1}, p_{2}\right)=$ $\int_{Z}\left|C D F_{p_{1}}(z)-C D F_{p_{2}}(z)\right| d z$, where $Z$ denotes the integration

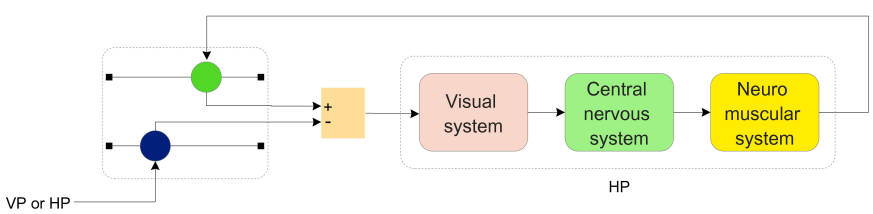

Fig. 3. Motor coordination between two players in the mirror game.

domain, and $C D F_{p_{i}}(z)$ denotes the cumulative distribution function of the distribution $p_{i}, i \in\{1,2\}$. Fig 2 (a) shows the position time series of the same HP and the corresponding PDF of velocity in two solo trials. It is visible that the two PDFs of velocity time series resemble each other in terms of their shape, and the EMD between them is 0.024. In contrast, the two PDFs of velocity time series in Fig.2(b) differ remarkably from each other, and the value of EMD is 0.604, which confirms the qualification of the PDF of velocity time series in solo trials as individual motor signature.

\section{Design of the Cognitive Architecture}

We design the cognitive architecture of the VP so as to replicate the main processes involved in making a human being play the mirror game (see Fig. 3). The visual system detects the ball's position on the string and generates visual signals, which are then transmitted to the central nervous system (CNS including brain and spinal cord). Several parts of the CNS (such as ventral horn, cerebellum and motor cortex) use an internal model to predict the kinematic characteristics of the other player's motion and generate the neural impulses that control the extension and contraction of muscles. Finally, the neuromuscular system activates and coordinates the muscles involved in generating the hand movements.

This architecture is mapped onto the real-time control schematics shown in Fig. 4 whose blocks are briefly described below.

- A camera is used to detect the position $r_{p}$ of the HP.

- A filtering and velocity estimation block is used to filter the position data acquired by the camera via a low pass filter and to estimate the velocity of the HP (reference) via the simple formula

$$
\hat{r}_{v}(t)=\frac{r_{p}\left(t_{k}\right)-r_{p}\left(t_{k-1}\right)}{T} \quad t \in\left[t_{k}, t_{k+1}\right]
$$

where $k \in N^{*}$, and $T=t_{k}-t_{k-1}$ denotes the sampling period of the camera. The estimated velocity is then used to predict the HP position over the next interval by using the expression:

$$
\hat{r}_{p}(t)=r_{p}\left(t_{k}\right)+\hat{r}_{v}(t)\left(t-t_{k}\right), \quad t \in\left[t_{k}, t_{k+1}\right]
$$

- At the core of the architecture lie the two blocks Temporal Correspondence Control and Signature Control. The former is designed to regulate the end effector model so that its motion tracks that of the HP with varying degrees of dynamic similarity. Specifically, it aims at minimizing the position error between the time series of the HP and 
that of the VP. The latter block uses the prerecorded velocity time series of a reference HP with the desired IMS (velocity profile) in order to generate the avatar trajectory with desired kinematic features. In particular, the aim of the signature controller is that of reducing the distance (computed in terms of EMD) between the velocity distribution of the VP and that of some reference HP it aims at replicating the motion characteristics of.

- The prerecorded velocity trajectory of a reference HP playing solo, representing the desired IMS, is stored in the Signature generator block while the signature of the avatar motion is estimated by the Signature estimation block.

- The end effector model is used to generate the avatar motion via an appropriate feedback control scheme. As mentioned before, the HKB equation is employed to describe the dynamics of the end effector.

- Finally, the output of the cognitive architecture (position $x$ and velocity $\dot{x}$ ) is used as reference motion for the VP.

In what follows we focus on the development of two different feedback control strategies that drive the cognitive architecture. Since our control algorithm is designed for a virtual computerized agent rather than a real robot platform, we do not take into account the effects of external disturbances as those mentioned in [28]. First, following the approach of [29], we develop an adaptive algorithm able to control the temporal correspondence between VP and HP during the game (green blocks in Fig 44). Note that adaptive control strategies are widely employed in a large variety of contexts, as for example those recently presented in [30], [31]. Then, we consider an optimal controller to solve simultaneously the multi objective control problem of tracking the trajectory of the HP while preserving the features of the desired IMS of interest (green and blue blocks in Fig44). For both strategies a proof of convergence is given before presenting numerical and experimental investigation of their performance.

\section{Adaptive Control of Temporal CORRESPONDENCE}

To solve the control problem of temporal correspondence, we propose an adaptive controller based on the end effector model shown in (2). Specifically, we choose the nonlinear controller given by

$$
u=\underbrace{\left[a(t)+b(t)\left(x-r_{p}\right)^{2}\right]\left(\dot{x}-\hat{r}_{v}\right)}_{\text {Coordination }}-\underbrace{C_{p} e^{-\delta\left(\dot{x}-\hat{r}_{v}\right)^{2}}\left(x-r_{p}\right)}_{\text {Temporal Correspondence }}
$$

where $r_{p}$ is the position of the HP, $\hat{r}_{v}$ is the estimated velocity, $C_{p}$ and $\delta$ are constant parameters, while the coupling parameters $a$ and $b$ are updated according to the adaptive laws:

$$
\dot{a}(t)=-e^{-2 a(t)}\left[\left(x-r_{p}\right)\left(y-\hat{r}_{v}\right)+\eta_{a}\left(x-r_{p}\right)^{2}\right]-\eta_{a}
$$

and

$$
\dot{b}(t)=\frac{y-\hat{r}_{v}}{e^{2 b(t)}}\left[\omega^{2} x+\left(\alpha y^{2}+\beta x^{2}-\gamma\right) y-\eta_{a}\left(y-\hat{r}_{v}\right)-u\right]-\eta_{a}
$$

where $\eta_{a}$ is a positive constant. Note that the control law (5) consists of two complementary terms. The former has the same structure as that of the coupling proposed in [20] to model the interaction between two HPs, albeit with the introduction of adaptive parameters to account for variability between different HPs. The second term, depending on the fixed parameters $C_{p}$ and $\delta$, deals with the position error when the velocity mismatch approaches zero and hence the first term decays to zero. When $\left|\dot{x}-\hat{r}_{v}\right|$ is relatively large, the coupling term of the HKB equation instead dominates and motor coordination between the two players becomes more pronounced during the mirror game.

Theoretical analysis of the adaptive control algorithm is given in what follows.

\section{A. Convergence analysis}

Theorem 4.1: The adaptive feedback controller (5) ensures that the solution of the controlled HKB model (2) satisfies

$$
\left|x(t)-r_{p}(t)\right| \leq e^{\eta_{a} T} \sqrt{\frac{2 \varepsilon}{e^{2 \eta_{a} T}-2}}+\frac{2}{e^{\eta_{a} T}} \sqrt{E(0)}, \quad t \in[0,+\infty)
$$

if $\eta_{a}$ is chosen so that $\eta_{a}>\frac{\ln 2}{2 T}$, where

$$
E(0)=\frac{1}{2}\left[\left(x(0)-r_{p}(0)\right)^{2}+\left(y(0)-\hat{r}_{v}(0)\right)^{2}+e^{2 a(0)}+e^{2 b(0)}\right]
$$

and

$$
\varepsilon=\sup _{k \in \mathbb{N}^{*}}\left(T^{2}+1\right)\left(\hat{r}_{v}(k T)-\hat{r}_{v}(k T-T)\right)^{2} .
$$

Proof: Choose the energy-like function

$$
E \triangleq \frac{1}{2}\left[\left(x-r_{p}\right)^{2}+\left(y-\hat{r}_{v}\right)^{2}+e^{2 a}+e^{2 b}\right]
$$

Note that $\hat{r}_{v}$ is fixed in each sampling interval $[k T,(k+$ 1)T), $k \in \mathbb{N}^{*}$. Then the time derivative of $E$ along the trajectories of (2) with $u$ defined in (5) is given by

$$
\begin{aligned}
\dot{E} & =\left(x-r_{p}\right)\left(\dot{x}-\hat{r}_{v}\right)+\left(y-\hat{r}_{v}\right) \dot{y}+e^{2 a} \dot{a}+e^{2 b} \dot{b} \\
& =\left(x-r_{p}\right)\left(y-\hat{r}_{v}\right)-\left(y-\hat{r}_{v}\right)\left[\left(\alpha y^{2}+\beta x^{2}-\gamma\right) y+\omega^{2} x-u\right] \\
& +\left(y-\hat{r}_{v}\right)\left[\omega^{2} x+\left(\alpha y^{2}+\beta x^{2}-\gamma\right) y-\eta_{a}\left(y-\hat{r}_{v}\right)-u\right]-\eta_{a} e^{2 b} \\
& -\left[\left(x-r_{p}\right)\left(y-\hat{r}_{v}\right)+\eta_{a}\left(x-r_{p}\right)^{2}\right]-\eta_{a} e^{2 a} \\
& =-\eta_{a}\left(x-r_{p}\right)^{2}-\eta_{a}\left(y-\hat{r}_{v}\right)^{2}-\eta_{a} e^{2 a}-\eta_{a} e^{2 b} \\
& =-2 \eta_{a} E, \quad t \in[k T,(k+1) T)
\end{aligned}
$$

Solving the above differential equation yields

$$
E(t)=e^{-2 \eta_{a}(t-k T)} E(k T), \quad t \in[k T,(k+1) T)
$$

Moreover, at the sampling point $k T$ we have

$$
\begin{aligned}
& E(k T)-E^{-}(k T) \\
= & \frac{1}{2}\left[\left(x-r_{p}(k T)\right)^{2}-\left(x-r_{p}(k T-T)-\hat{r}_{v}(k T-T) T\right)^{2}\right. \\
+ & \left.\left(y-\hat{r}_{v}(k T)\right)^{2}-\left(y-\hat{r}_{v}(k T-T)\right)^{2}\right] \\
\leq & \left(r_{p}(k T)-r_{p}(k T-T)-\hat{r}_{v}(k T-T) T\right)^{2} \\
+ & \left(\hat{r}_{v}(k T)-\hat{r}_{v}(k T-T)\right)^{2}+E^{-}(k T) \\
= & \left(1+T^{2}\right)\left(\hat{r}_{v}(k T)-\hat{r}_{v}(k T-T)\right)^{2}+E^{-}(k T)
\end{aligned}
$$

which is equivalent to

$$
E(k T) \leq \varepsilon+2 E^{-}(k T)
$$




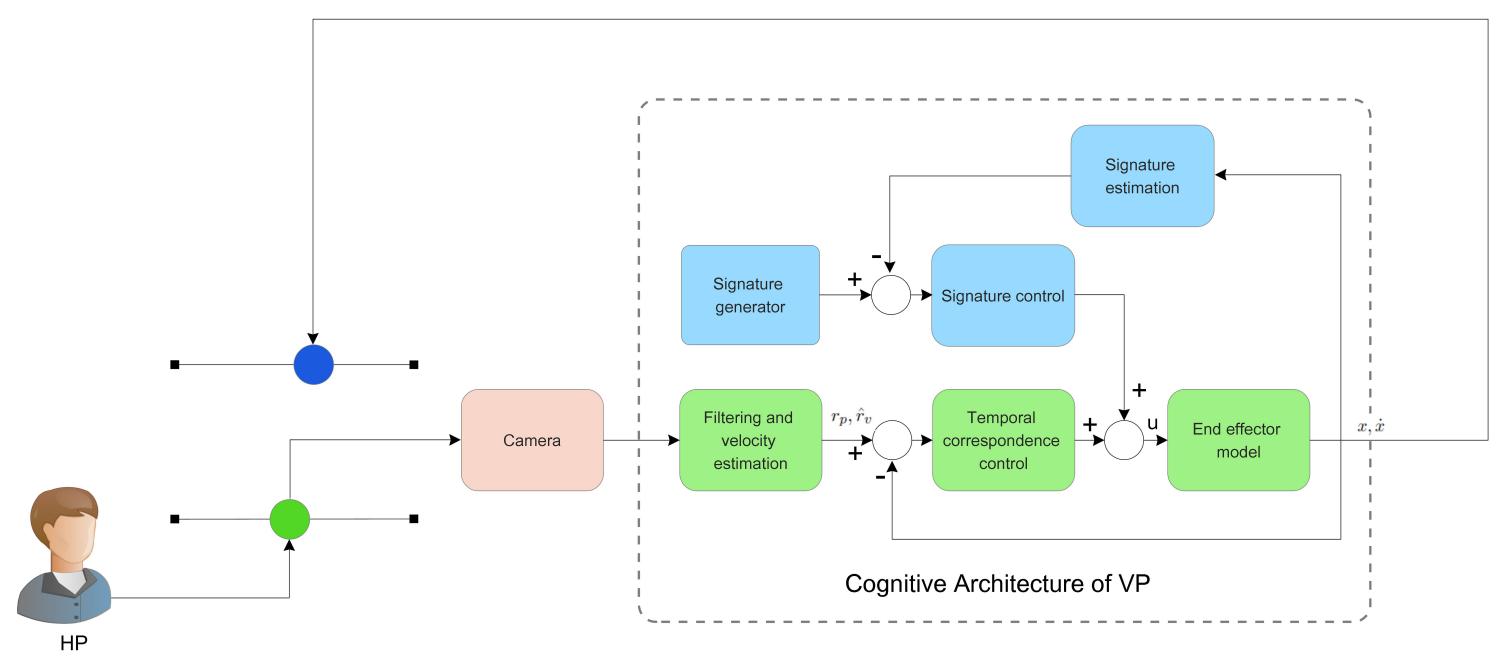

Fig. 4. Block diagram of the cognitive architecture of VP. Green blocks allow for the control of temporal correspondence between the VP and the HP, and blue blocks take into account the desired individual motor signature.

where $\varepsilon=\sup _{k \in \mathbb{N}^{*}}\left(T^{2}+1\right)\left(\hat{r}_{v}(k T)-\hat{r}_{v}(k T-T)\right)^{2}$ and $E^{-}(k T)=\lim _{t \rightarrow k T} E(t)$. Evaluating $(9)$ and $(10)$ at $t=(k+1) T$ and nesting the inequalities backwards till $t=0$, we get

$$
\begin{aligned}
E(k T) & \leq \varepsilon\left[1+\frac{2}{e^{2 \eta_{a} T}}+\left(\frac{2}{e^{2 \eta_{a} T}}\right)^{2}+\ldots+\left(\frac{2}{e^{2 \eta_{a} T}}\right)^{k-1}\right] \\
& +\left(\frac{2}{e^{2 \eta_{a} T}}\right)^{k} E(0)=\varepsilon \frac{1-\left(\frac{2}{e^{2} \eta_{a} T}\right)^{k}}{1-\frac{2}{e^{2 \eta_{a} T}}}+\left(\frac{2}{e^{2 \eta_{a} T}}\right)^{k} E(0) \\
& \leq \frac{\varepsilon}{1-\frac{2}{e^{2 \eta_{a} T}}}+\frac{2}{e^{2 \eta_{a} T}} E(0), \quad \forall k \in \mathbb{N}^{*}
\end{aligned}
$$

when the inequality $\eta_{a}>\frac{\ln 2}{2 T}$ holds. Moreover, combining 9 with (11), we get

$$
E(t) \leq \frac{\varepsilon}{1-\frac{2}{e^{2 \eta_{a} T}}}+\frac{2}{e^{2 \eta_{a} T}} E(0), \quad t \in[0,+\infty)
$$

which clearly implies

$$
\left|x(t)-r_{p}(t)\right| \leq e^{\eta_{a} T} \sqrt{\frac{2 \varepsilon}{e^{2 \eta_{a} T}-2}}+\frac{2}{e^{\eta_{a} T}} \sqrt{E(0)}, \quad t \in[0,+\infty)
$$

Remark 4.1: It is easy to demonstrate that the coupling parameters $a$ and $b$ are upper bounded with the proposed adaptive laws.

Remark 4.2: Since $r_{p}(t) \in[0, l], \forall t \geq 0$ and $\left|\hat{r}_{v}(t)\right| \leq \frac{l}{T}$, the following inequality holds

$$
\varepsilon=\sup _{k \in \mathbb{N}^{*}}\left(T^{2}+1\right)\left(\hat{r}_{v}(k T)-\hat{r}_{v}(k T-T)\right)^{2} \leq \frac{4 l^{2}\left(1+T^{2}\right)}{T^{2}}
$$

where $l$ refers to the length of the string. Generally, the upper bound for the position error is relatively conservative. When the velocity of the HP is small, $\varepsilon$ is small as well, and the estimation for the position error is accurate enough. In addition, taking the limit of (11) as $k T \rightarrow \infty$ and combining it with $(8)$, the position error between the two players satisfies the following inequality:

$$
\limsup _{k T \rightarrow+\infty}\left|x(t)-r_{p}(t)\right| \leq e^{\eta_{a} T} \sqrt{\frac{2 \varepsilon}{e^{2 \eta_{a} T}-2}}
$$

Similarly, we can estimate the velocity error as

$$
\left|\dot{x}(t)-\hat{r}_{v}(t)\right| \leq e^{\eta_{a} T} \sqrt{\frac{2 \varepsilon}{e^{2 \eta_{a} T}-2}}+\frac{2}{e^{\eta_{a} T}} \sqrt{E(0)}, \quad t \in[0,+\infty)
$$

While being effective in achieving bounded tracking of the HP position, the control approach derived so far is unable to explicitly guarantee that the generated motion follows a desired IMS. Therefore we consider a different scheme based on optimal control.

\section{Optimal Temporal CoRrespondence And SignATURE CONTROL}

The second approach we propose is based on optimal control, which is an effective framework to allow for movement coordination and reconcile target tracking and individual motor signature [32]. We assume that in the mirror game the motion of the VP can be decomposed into a series of goal-directed movements (see Fig. 5) influenced by both the position of the HP and the desired individual motor signature. Thus, we formulate the problem of driving the end effector motion as described by $[2]$ on a finite time interval $\left[t_{k}, t_{k+1}\right]$ as the dynamic optimization problem

$$
\min _{u \in R} J
$$

where

$$
\begin{aligned}
J & =\frac{\theta_{p}}{2} \underbrace{\left(x\left(t_{k+1}\right)-\hat{r}_{p}\left(t_{k+1}\right)\right)^{2}}_{\text {Temporal Correspondence }} \\
& +\frac{1}{2} \int_{t_{k}}^{t_{k+1}} \theta_{\sigma} \underbrace{\left(\dot{x}(\tau)-r_{\sigma}(\tau)\right)^{2}}_{\text {Similarity }}+\eta_{m} u(\tau)^{2} d \tau
\end{aligned}
$$




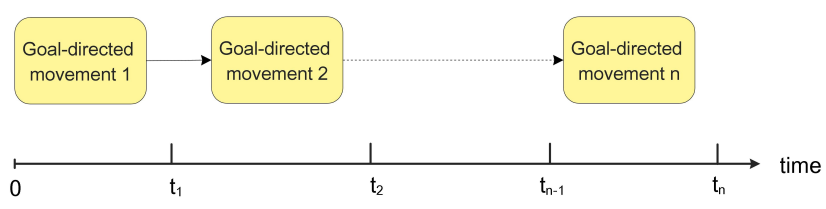

Fig. 5. Movement of the VP end effector in the mirror game

with the constraint $\theta_{p}+\theta_{\sigma}=1$ and $\theta_{p}, \theta_{\sigma}, \eta_{m}>0$ being tunable control parameters. Here, $\hat{r}_{p}\left(t_{k+1}\right)$ denotes the estimated position of the HP at time $t_{k+1}$ (see (4) for further details), while $r_{\sigma}$ refers to a prerecorded velocity time series representing the desired motor signature. For the sake of simplicity, the optimization interval $\left[t_{k}, t_{k+1}\right]$ in the cost function (13) corresponds to the sampling interval. The above cost function mainly consists of three terms. The first term aims at minimizing the mismatch between the position time series of the HP and that of the VP. The second term takes care of making the velocity profile of the VP as close as possible to the reference one (motor signature). The last term guarantees boundedness of the control effort. In particular, the idea behind this cost function is that the human-like movement of the VP emerges from the integration of three different goals related to temporal correspondence, motor signature and control energy expenditure, respectively. Note that the VP acts as a leader when $\theta_{p}$ is close to 0 , since the term related to the position error $x-\hat{r}_{p}$ in the cost function is negligible and the only aim of the VP is to exhibit the motion characterized by the desired motor signature. On the other hand, the VP behaves as a follower if $\theta_{p}$ is close to 1 as in this case the controller aims solely at minimizing the mismatch between the HP and VP terminal positions. For small values of $\eta_{m}$, the control energy can be large enough for the VP to achieve the desired tracking performance or signature matching. On the other hand, for larger values of $\eta_{m}$ the control energy is small, which results into a poorer performance of the VP.

\section{A. Convergence Analysis}

To prove stability of the optimal control algorithm, we focus on proving boundedness of the position error between the reference input and the output of the cognitive architecture. In particular, optimality of the cost function is guaranteed in each optimization interval if the damped harmonic oscillator is adopted as end effector model instead of the HKB oscillator. Since both the reference position $r_{p}$ and the desired velocity $r_{\sigma}$ are bounded, we assume $r_{p} \in[\underline{r}, \bar{r}]$ and $r_{\sigma} \in[\underline{v}, \bar{v}]$.

Theorem 5.1: The optimal control algorithm applied to the HKB oscillator (2) with cost function (13) ensures bounded position error between HP and VP.

Proof: First of all, we need to demonstrate that there exists a limit cycle in the HKB oscillator

$$
\left\{\begin{array}{l}
\dot{x}=y \\
\dot{y}=-\left(\alpha x^{2}+\beta y^{2}-\gamma\right) y-\omega^{2} x
\end{array}\right.
$$

Choose the energy-like function as $V(x, y)=\frac{\omega^{2} x^{2}+y^{2}}{2}$. The time derivative of $V(x, y)$ along the trajectory of the HKB oscillator

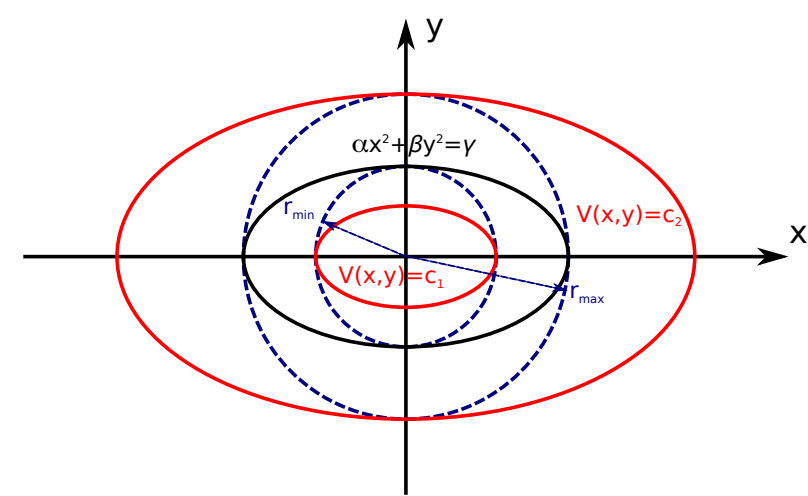

Fig. 6. Illustration on the construction of region $\Re$. The black ellipse is described by the equation $\alpha x^{2}+\beta y^{2}=\gamma$, and the region $\Re$ refers to the ringshaped area bounded by two red ellipses corresponding to $V(x, y)=c_{1}$ and $V(x, y)=c_{2}$, respectively.

(14) is given by

$$
\begin{aligned}
\dot{V}(x, y) & =\omega^{2} x \dot{x}+y \dot{y}=\omega^{2} x y-\left(\alpha x^{2}+\beta y^{2}-\gamma\right) y^{2}-\omega^{2} x y \\
& =-\left(\alpha x^{2}+\beta y^{2}-\gamma\right) y^{2}
\end{aligned}
$$

Define

$$
r_{\text {max }}:=\max \left(\sqrt{\frac{\gamma}{\alpha}}, \sqrt{\frac{\gamma}{\beta}}\right), r_{\text {min }}:=\min \left(\sqrt{\frac{\gamma}{\alpha}}, \sqrt{\frac{\gamma}{\beta}}\right)
$$

and construct a region $\mathfrak{R}$ as follows (see Fig. 6 )

$$
\mathfrak{R}:=\left\{(x, y) \in \mathbb{R}^{2}: c_{1} \leq V(x, y) \leq c_{2}\right\}
$$

where the positive constants $c_{1}$ and $c_{2}$ satisfy

$$
r_{\text {min }}=\max \left(\sqrt{\frac{2 c_{1}}{\omega^{2}}}, \sqrt{2 c_{1}}\right), r_{\text {max }}=\min \left(\sqrt{\frac{2 c_{2}}{\omega^{2}}}, \sqrt{2 c_{2}}\right)
$$

Clearly, $\mathfrak{R}$ contains no stationary points of the system. Indeed, the only stationary point of the system is $(x, y)=(0,0)$, which is located outside the region $\mathfrak{R}$. Moreover, $\dot{V}(x, y) \geq 0$ when $V(x, y)=c_{1}$ and $\dot{V}(x, y) \leq 0$ when $V(x, y)=c_{2}$. According to the Poincare-Bendixson theorem, we conclude that the HKB oscillator (14) has a limit cycle in $\mathfrak{R}$.

Let $J^{*}$ denote the value of the cost function $(13)$ with the optimal control algorithm in each time interval, and let $J_{0}$ represent the value of the corresponding cost function when $u=0$. Since $u$ aims at minimizing the value of the cost function for all $k \in \mathbb{N}^{*}$, we can write

$J^{*} \leq J_{0}=\frac{\theta_{p}}{2}\left(x\left(t_{k+1}\right)-\hat{r}_{p}\left(t_{k+1}\right)\right)^{2}+\frac{\theta_{\sigma}}{2} \int_{t_{k}}^{t_{k+1}}\left(\dot{x}(\tau)-r_{\sigma}(\tau)\right)^{2} d \tau$

Since $r_{p}$ is bounded, $\hat{r}_{p}\left(t_{k+1}\right)$ is bounded as well according to (3) and (4). In addition, also $r_{\sigma}(\tau)$ is bounded, and so are $x(t)$ and $\dot{x}(t)$ as the trajectory of the HKB oscillator converges to the limit cycle in $\mathfrak{R}$. Thus, we can claim that $J_{0}$ is bounded for $k \in \mathbb{N}^{*}$, which implies boundedness of $J^{*}$ and as a consequence of the position error between VP and HP.

Remark 5.1: It is demonstrated that the bound on the tracking error $\left|x\left(t_{k+1}\right)-\hat{r}_{p}\left(t_{k+1}\right)\right|$ converges to 0 as $\theta_{p} \rightarrow 1$ and $\eta_{m} \rightarrow 0$. Similarly, the bound of the velocity error $\mid \dot{x}\left(t_{k+1}\right)-$ 
$r_{\sigma}\left(t_{k+1}\right) \mid$ goes to 0 if $\theta_{\sigma} \rightarrow 1, \eta_{m} \rightarrow 0$ and $r_{\sigma}\left(t_{k}\right)=y\left(t_{k}\right)$ (see Supplementary Material for the detailed analysis).

The analytical solution to the optimization problem (12) is available if a linear damped harmonic oscillator is employed as end effector model, and Pontryagin's minimum principle provides necessary and sufficient conditions to solve the minimization problem [33].

Corollary 5.1: Given the linear system $\ddot{x}+a \dot{x}+b x=u$, the optimal control approach guarantees convergence to the optimum solution over each subinterval.

Proof: According to the fundamental theorem of the calculus of variations, we need to examine the second variation of the given cost function in order to establish the optimum. From the conclusions in [33], the second variation of the cost function (13) is given by

$$
\begin{aligned}
\delta^{2} J & =\theta_{p}\left[\delta x\left(t_{k+1}\right)\right]^{2} \\
& +\int_{t_{k}}^{t_{k+1}}\left(\begin{array}{ll}
\delta X & \delta u
\end{array}\right)\left(\begin{array}{cc}
H_{X X} & H_{X u} \\
H_{u X}^{T} & H_{u u}
\end{array}\right)\left(\begin{array}{c}
\delta X \\
\delta u
\end{array}\right) d t
\end{aligned}
$$

where $H$ is the Hamiltonian

$$
H(X, u, \lambda)=\frac{1}{2} \theta_{s}\left(\dot{x}-r_{\sigma}\right)^{2}+\frac{1}{2} \eta u^{2}+\lambda^{T}\left(\begin{array}{c}
y \\
-a y-b x+u
\end{array}\right)
$$

with $X=[x, \dot{x}]^{T}=[x, y]^{T}$ and $\lambda=\left[\lambda_{1}, \lambda_{2}\right]^{T}$. Rewrite the linear system in matrix form $\dot{X}=A X+B u$, where

$$
A=\left(\begin{array}{cc}
0 & 1 \\
-b & -a
\end{array}\right), \quad B=\left(\begin{array}{l}
0 \\
1
\end{array}\right)
$$

Let $X=X^{*}+\delta X$ and $u=u^{*}+\delta u$, where $X^{*}$ and $u^{*}$ denote optimal state and optimal control, respectively. Since $\dot{X}^{*}=$ $A X^{*}+B u^{*}$, we get

$$
\dot{\delta} X=A \delta X+B \delta u
$$

where $\delta X=[\delta x, \delta \dot{x}]^{T}$. Thus, it follows from $H_{X u}=H_{u X}=$ $\left[\begin{array}{ll}0 & 0\end{array}\right]^{T}, H_{u u}=\eta>0$ and

$$
H_{X X}=\left(\begin{array}{cc}
0 & 0 \\
0 & \theta_{s}
\end{array}\right) \geq 0
$$

that

$$
\begin{aligned}
\delta^{2} J & =\theta_{p}\left[\delta x\left(t_{k+1}\right)\right]^{2}+\int_{t_{k}}^{t_{k+1}} \delta X(t)^{T} H_{X X} \delta X(t)+\eta(\delta u(t))^{2} d t \\
& =\theta_{p}\left[\delta x\left(t_{k+1}\right)\right]^{2}+\int_{t_{k}}^{t_{k+1}} \theta_{s}(\delta \dot{x}(t))^{2}+\eta(\delta u(t))^{2} d t \geq 0
\end{aligned}
$$

Moreover, $\delta^{2} J=0$ is equivalent to $\delta x\left(t_{k+1}\right)=0, \delta \dot{x}(t)=0$ and $\delta u(t)=0$ for all $t \in\left[t_{k}, t_{k+1}\right]$, which yields $\delta x(t)=\delta x\left(t_{k}\right)=0$ from (15). This corresponds to the optimal solution $X^{*}$ and the optimal control $u^{*}$. Therefore, we conclude that the optimal control ensures the minimum value of the cost function (13) for the linear system in each time interval.

\section{VALIDATION}

In this section we experimentally validate our control algorithms on a simple, yet effective, set-up implemented at the University of Bristol, UK. Experimental data of human-human interaction is used to evaluate the matching performance of the virtual player, and a comparison with existing VP models is provided as well.

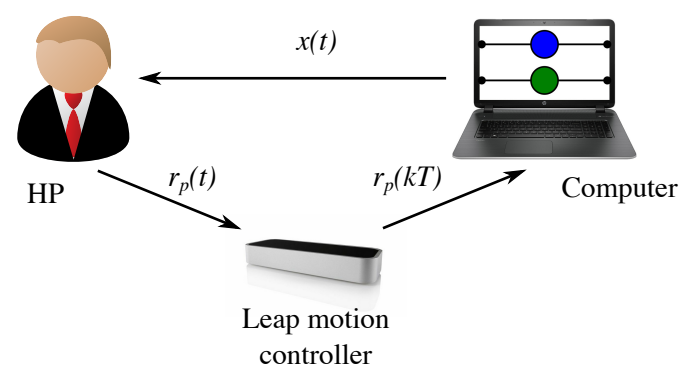

Fig. 7. Experimental set-up of the mirror game between a HP and a VP. The position of the human fingertip $r_{p}(t)$ is detected by a leap motion controller, and the sampled position $r_{p}(k T)$ is sent to the computer, while the position $x(t)$ of the VP is generated by implementing the control algorithm. Two balls are shown on the computer screen, which describe the end effector positions of the HP (green ball) and the VP (blue ball), respectively.

\section{A. Experimental Set-up}

The employed set-up was developed for measuring motions of players in the one-dimensional mirror game. A human participant is required to join the game while interacting with a VP (implemented on a laptop computer). In order to detect the position of her/his hand, a leap motion controller [34] is employed (see Fig. 77). The leap motion controller and the laptop computer are both placed on a table whose height is around $70 \mathrm{~cm}$. The HP is required to wave her/his hand horizontally over the leap motion controller at a vertical distance of approximately $50 \mathrm{~cm}$. Indeed, at this distance the horizontal resolution of the device is maximum and it is able to measure the hand position within a range of $60 \mathrm{~cm}$. The position of the hand of the HP within this interval is mapped into the interval $[-0.5,0.5]$ and visualized on the computer screen as a green solid circle, while the position of the VP is visualized as a blue solid circle. Players can be either standing or seated. After the game is initialized, there is a 2s wait before data recording begins and the game starts. This initial delay is used to allow the HP to place her/his hand over the leap motion controller. Human players are not instructed before playing the game, but they are just told to lead or follow a VP during 60s rounds.

Remark 6.1: The real-time implementation of adaptive (Section IV) and optimal (Section V) control in Matlab can be expensive. Specifically, the computational burden and the time required to implement such control strategies mainly depend on the numerical method employed to integrate differential equations (adaptive control), and on the desired accuracy of the numerical solutions (optimal control). In our case we adopt the simple Euler method to numerically integrate differential equations, and we choose the value (RelTol, 1e-1) as numerical accuracy for the function "bvp4c" employed to solve the twopoint boundary problem. We find that with this choice of algorithm, Matlab is fast enough to solve the problem in realtime.

\section{B. Measures}

The temporal correspondence between the VP and the HP is evaluated according to the following indexes: root mean square 
(RMS) of the position mismatch, relative position error (RPE), circular variance $(\mathrm{CV})$ and time lag $(\mathrm{TL})$.

1) RMS: The root mean square of the position error between two players describes the tracking accuracy of the follower in the mirror game:

$$
R M S=\sqrt{\frac{1}{n} \sum_{i=1}^{n}\left(x_{1, i}-x_{2, i}\right)^{2}}
$$

where $n$ is the number of sampling steps in the simulation, and $x_{1, i}$ and $x_{2, i}$ denote the positions of leader and follower at the $i$-th sampling step, respectively.

2) RPE: The relative position error is a measure of how well the follower tracks the leader [16]. Positive values of RPE indicate that the follower is indeed behind the leader:

$$
R P E=\left\{\begin{array}{c}
\left(x_{1}(t)-x_{2}(t)\right) \operatorname{sgn}\left(\dot{x}_{1}(t)\right), \\
\text { if } \operatorname{sgn}\left(\dot{x}_{1}(t)\right)=\operatorname{sgn}\left(\dot{x}_{2}(t)\right) \neq 0 \\
\left|x_{1}(t)-x_{2}(t)\right|, \text { otherwise. }
\end{array}\right.
$$

where $x_{1}(t)$ and $x_{2}(t)\left(\dot{x}_{1}(t)\right.$ and $\left.\dot{x}_{2}(t)\right)$ are positions (velocities) of the leader and follower at time $t$, respectively.

3) CV: The circular variance is used to quantify the coordination level between two players

$$
C V=\left\|\frac{1}{n} \sum_{k=1}^{n} e^{i \Delta \Phi_{k}}\right\| \quad \in[0,1]
$$

where $\Delta \Phi_{k}$ represents the relative phase between them at the $k$-th sampling step, $n$ refers to the total number of time steps and $\|\cdot\|$ denotes the 2-norm [35].

4) TL: The time lag describes the shifted time that achieves the maximum cross-covariance of two time series. It is sensitive to the changes of motion direction, hence can be interpreted as the average reaction time of the player in the mirror game [36].

\section{Results}

1) VP driven by the ICA based on adaptive control: The parameters for the HKB equation and the adaptive feedback controller (AFC) in (5) are set heuristically as follows: $\alpha=10$, $\beta=20, \gamma=-1, \omega=0.1, a(0)=-5, b(0)=-5, C_{p}=40$ and $\delta=0.25$. In our implementation the sampling time is $T=0.1 \mathrm{~s}$ and therefore $\eta_{a}=30>\frac{\ln 2}{2 T} \simeq 0.35$. In particular, the values of all the previous parameters have been chosen so that the response of the HKB oscillator to several sinusoidal signals with different frequencies was qualitatively the same as that of a HP trying to track the same references. Moreover, it is worthy of note that the initial values of $a$ and $b$ influence the performance of the avatar only at start-up.

The reactive-predictive controller (RPC) proposed in [2] is also implemented to compare its performance against that of our adaptive feedback controller when considering the same input trajectory from a human leader. Following the scheme presented in [2] to implement the RPC, the dynamics of the VP is described by $\ddot{x}=\sum_{i=1}^{5} A_{i} \omega_{i} \cos \left(\omega_{i} t\right)+f$, where $x \in \mathbb{R}$

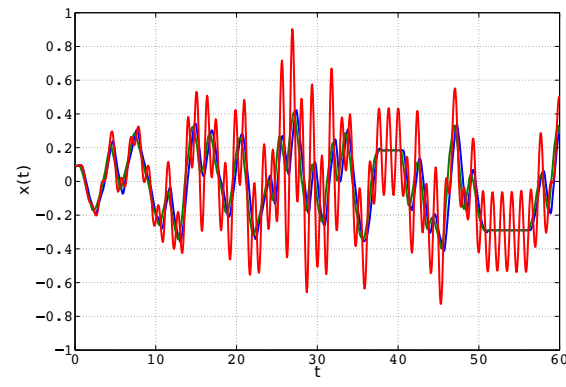

(a) position time series

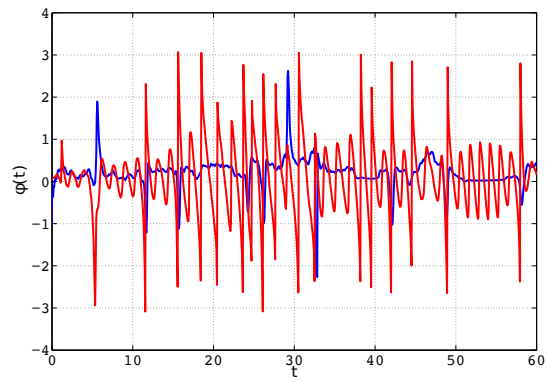

(b) relative phase time series

Fig. 8. Time series of position (a) and relative phase (b) between human leader and virtual follower; blue (AFC), red (RPC), green (human leader)

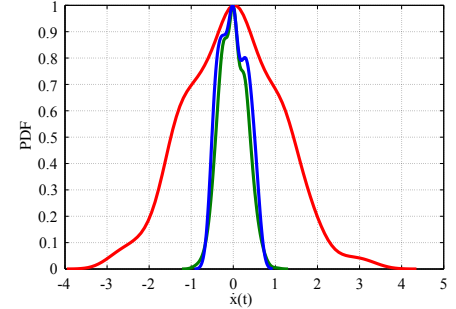

(a) veocity $\mathrm{PDF}$

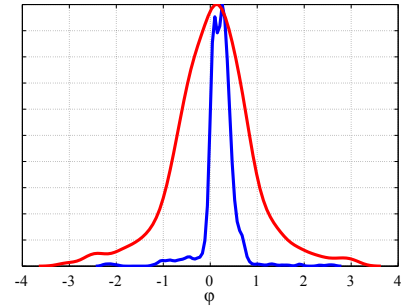

(b) relative phase PDF
Fig. 9. Distributions of velocity (a) and relative phase (b) between human leader and virtual follower; blue (AFC), red (RPC), green (human leader)

represents the position of the avatar and $\dot{f}=k\left(\hat{r}_{v}-\dot{x}\right), \quad k>0$, with parameters $A_{i}$ being estimated adaptively as

$$
\dot{A}_{i}=\lambda\left[\hat{r}_{v}-\sum_{i=1}^{5} A_{i} \sin \left(\omega_{i} t\right)\right] \sin \left(\omega_{i} t\right), \quad \lambda>0
$$

As suggested in [2], in this case the parameters are chosen as follows: $\omega_{1}=0.025, \omega_{2}=0.05, \omega_{3}=0.075, \omega_{4}=0.1, \omega_{5}=$ $0.125, \lambda=0.01, k=30$ and $A_{i}(0)=0, \forall i=1, \ldots, 5$.

To compare the performance of the two algorithms, we plot the time series of both position and relative phase (see Fig. 8) together with the distributions of velocity and relative phase of HP and VP (see Fig. 9). In particular, the relative phase between the two players is defined as $\Delta \phi=\phi_{H P}-\phi_{V P}$, where $\phi_{H P}$ and $\phi_{V P}$ are the phases of human and virtual player, respectively. Specifically, the phase is estimated according to the method proposed in [37]. Note that positive values of $\Delta \phi$ correspond to the avatar following the HP during the game. 

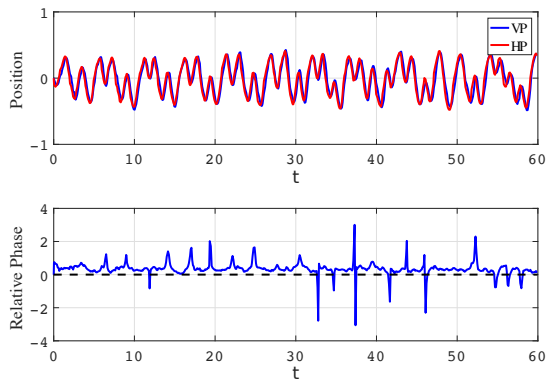

(a) Temporal Correspondence

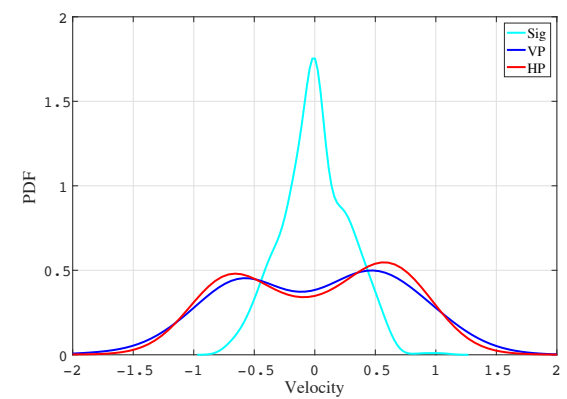

(b) Similarity

Fig. 10. Time evolution of positions and relative phase (a) and PDF of velocities (b) while the VP is driven by the optimal control and acts as follower in the mirror game

We observe that, when using the reactive-predictive controller, the position of the VP presents oscillations away from the human participant position not only when s/he is moving, but also when s/he is still. Such an oscillatory feature does not appear when using the adaptive feedback controller. In general, both the position error $e=x-r_{p}$ and the velocity error $\dot{e}=\dot{x}-\hat{r}_{v}$ are higher when using the RPC strategy. When using the adaptive feedback controller, the position error remains smaller never exceeding 0.2 , while it can become as high as 0.8 when using RPC. Similarly, the velocity error never exceeds 0.62 for the AFC, while it goes up to a maximum of 3 for the RPC.

Moreover, when using AFC, the relative phase time series is much closer to 0 than that obtained when using RPC, meaning that with our proposed algorithm it is possible for the VP to better synchronize with the human leader. Such results are confirmed by the relative phase distributions obtained when using both the algorithms, as shown in Fig. 9 (b). Finally, the difference in the velocity distributions of HP and VP is much more evident when RPC is used, confirming that our strategy better captures the features of the HP and is therefore able to replicate more accurately the kinematic properties observed in human motor coordination in the context of the mirror game.

2) VP driven by the ICA based on optimal control: The parameters of the VP are set heuristically as follows: $\alpha=1$, $\beta=1, \gamma=1, \omega=1, \eta_{m}=10^{-4}$ and the sampling period $T=t_{k+1}-t_{k}=0.03 \mathrm{~s}$. In order for the VP to play the mirror game as a follower, we set the control parameters $\theta_{p}=0.9$ and $\theta_{\sigma}=0.1$, which makes the VP play in a follower configuration (minimizing the position mismatch more than
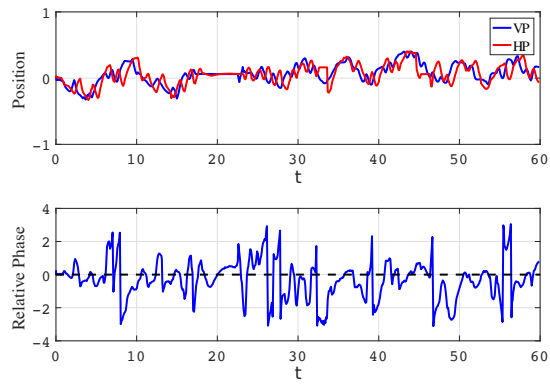

(a) Temporal Correspondence

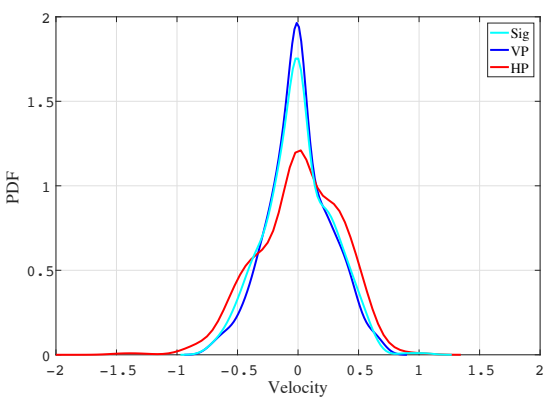

(b) Similarity

Fig. 11. Time evolution of positions and relative phase (a) and PDF of velocities (b) while the VP is driven by the optimal control and acts as leader in the mirror game

the signature EMDs). As shown in Fig 10(a), the VP performs quite well as a follower during the game; indeed, the root mean square (RMS) of the position error is equal to 0.089 . In order to distinguish the leader from the follower in the game, we also calculate the relative phase between HP and VP. From the bottom panel in Fig. 10 (a) we observe that the majority of relative phase is positive (with a mean value of 0.35 ), meaning that the VP is following the HP in the game for most of the time. The circular variance $(\mathrm{CV})$ is also calculated to quantify the coordination level between two players. The CV between HP and VP is 0.94, which indicates a high coordination level. As for the distribution of the velocity, Fig. 10(b) shows that the VP signature (blue line) is closer to that of the HP (red line) than the desired motor signature (cyan line). This is due to the choice of the control parameters in the cost function (13) that render the strategy able to minimize more the position error between the players. The measured EMDs at the end of the trial are given as follows: $\operatorname{EMD}($ Sig,$H P)=\operatorname{EMD}($ Sig,$V P)=0.05$ and $\operatorname{EMD}(V P, H P)=0.008$.

The VP can be enabled to play the game as a leader by changing the control parameters setting $\theta_{p}=0.1$ and $\theta_{\sigma}=0.9$. Experimental results are shown in Fig. 11. The RMS of the tracking error is 0.12 , and the $\mathrm{CV}$ between the two players is 0.56. As depicted in the bottom panel of Fig. 11 (a), the majority of the relative phase time series is negative (with a mean value of -0.31 ), meaning that now the VP is leading the $\mathrm{HP}$ during the game for most of the time. In contrast to the previous case, the velocity distributions shown in Fig. 11.b) 

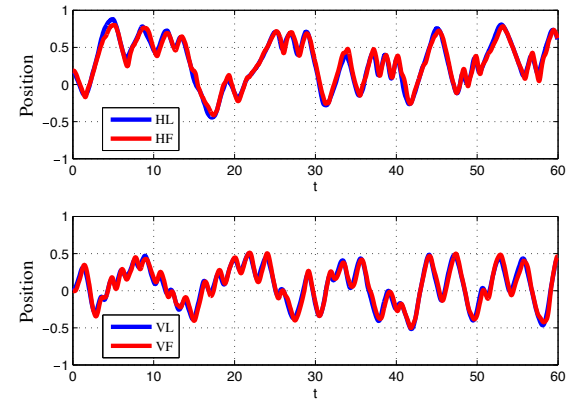

Fig. 12. Position time series of the HP pair (upper panel) and the corresponding VP pair (lower panel) in the mirror game.

confirm that the VP is now matching well the desired signature (velocity profile): $\operatorname{EMD}(S i g, H P)=\operatorname{EMD}(V P, H P)=0.01$ and $\operatorname{EMD}($ Sig,$V P)=0.002$.

The tracking performance in Fig. 10.a) is superior to that shown in Fig. 11 (a) in terms of coordination level and tracking error, which indicates that the VP is more accurate at tracking the leader's trajectories than the HP. Moreover, the relative phase is mostly positive as shown in Fig. 10(a) as opposed to the mainly negative relative phase in Fig. 11(a), which is consistent with the role (follower or leader) assigned to the VP. Moreover, the velocity PDF of the virtual player is close to that of the human player when the former is driven to track the motion of the latter, as shown in Fig. 10(b); vice versa, it is close to the desired motor signature, as shown in Fig. 111(b). This confirms that the VP succeeds in acting as follower or leader as desired with the proposed control approach.

3) Interaction between two VPs: As mentioned before, our final goal is to create a customized VP able to "replicate" the kinematic features of a given HP in the mirror game. To test how well the VP can replicate the features of a given HP, we carried out the following experiment. First of all, two HPs are required to play the mirror game in a Leader-Follower condition. Then the signatures of the human leader (HL) and the human follower (HF) are fed into a virtual leader (VL) and a virtual follower (VF), respectively. Finally, we make the VL $\left(\theta_{p}=0.43\right)$ and the VF $\left(\theta_{p}=0.92\right)$ play the mirror game together. In Fig. 12, the upper panel shows the time evolution of the position trajectories of the $\mathrm{HL}$ and the HF, while the lower panel presents those of the corresponding VL and VF. It appears that the VL and the VF succeed in matching the kinematic characteristics of the HL and the HF in terms of the RMS value of their position error $[\operatorname{RMS}(H L, H F)=0.0466$ and $\operatorname{RMS}(V L, V F)=0.0497]$ and the time lag between the two players $[\mathrm{TL}(H L, H F)=0.09$ and $\operatorname{TL}(V L, V F)=0.09]$. In addition, Table $\mathrm{I}$ gives the values of EMDs and describes the matching results quantitatively. $\mathrm{Sig}_{L}$ and $\mathrm{Sig}_{F}$ represent the signatures of HL and HF when playing solo, respectively. It shows that the proposed approach allows to replicate effectively the game dynamics between two humans playing the mirror game via two coupled VPs.

4) Comparison with existing models: In order to compare different VP models, following the approach of [26] we need to establish a benchmark, which describes the general kine-
TABLE I

MATCHING PERFORMANCE OF VPS IN TERMS OF EMD.

\begin{tabular}{|c|c|c|c|}
\hline EMD $\left(\right.$ Sig $\left._{L}, \mathrm{HL}\right)$ & 0.010 & $\mathrm{EMD}\left(\right.$ Sig $\left._{F}, \mathrm{HF}\right)$ & 0.007 \\
\hline $\mathrm{EMD}\left(\mathrm{Sig}_{L}, \mathrm{VL}\right)$ & 0.006 & $\mathrm{EMD}\left(\right.$ Sig $\left._{F}, \mathrm{VF}\right)$ & 0.006 \\
\hline EMD(HL,HF $)$ & 0.0034 & $\mathrm{EMD}(\mathrm{VL}, \mathrm{HL})$ & 0.0052 \\
\hline EMD(VL,VF) & 0.0031 & $\mathrm{EMD}(\mathrm{VF}, \mathrm{HF})$ & 0.0053 \\
\hline
\end{tabular}

TABLE II

INDEXES OF TEMPORAL CORRESPONDENCE

\begin{tabular}{|c|c|c|c|c|c|}
\hline & HPs & OPC & HKB & RPC & JKE \\
\hline RPE & 0.0914 & 0.0816 & 2.1767 & 0.3838 & 0.1467 \\
\hline CV & 0.3011 & 0.1002 & 0.9400 & 0.7602 & 0.4859 \\
\hline TL & 0.2035 & 0.1274 & 1.5192 & 0.0428 & -0.9674 \\
\hline
\end{tabular}

matic characteristics of human participants in the mirror game. To this aim, 5 HPs were asked to track a prerecorded reference signal, and indexes of temporal correspondence were recorded to represent a benchmark of typical human dynamics. The existing VP models were then enabled to track the same reference. Corresponding indexes were computed for the VPs and compared with the benchmark to evaluate the proposed control algorithms. We adopted the following models to drive the VP: our strategy based on optimal control (OPC), Haken-KelsoBunz model (HKB)[18], reactive-predictive control (RPC)[2] and Jirsa-Kelso excitator (JKE) [7]. The parameter setting of existing VP models is the same as that in the relevant references. In Table II], we show the benchmark of temporal correspondence and performance indexes of the corresponding VP models. On the whole, our algorithm performs best in terms of matching the benchmark among all the VP models. In addition to temporal correspondence, we also computed how similar the VP signature is to that of the HP whose trajectory was used as prerecorded reference signal during the benchmark experiment (see $\mathrm{Fig} 13$ ). We find again that the optimal control strategy developed in this paper is the best in terms of replicating the human-like movement with an estimated $\operatorname{EMD}(H P, O P C)=0.0184$.

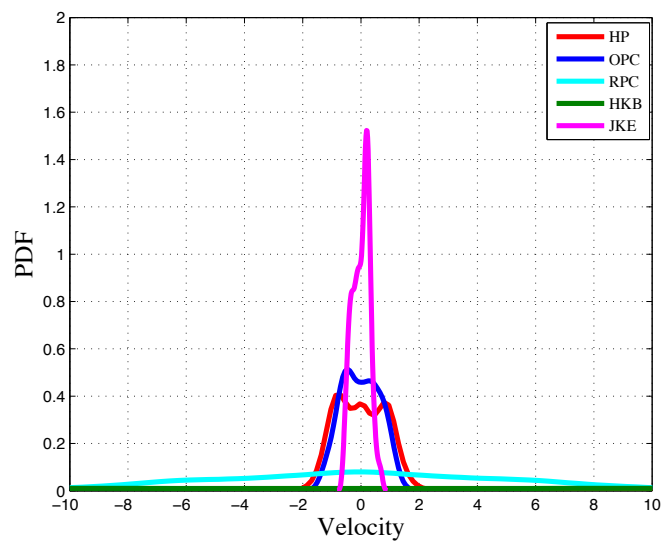

Fig. 13. PDF of velocity time series for different VP models. 


\section{CONCLUSIONS}

We presented the novel design of an interactive cognitive architecture able to drive a virtual player to play the mirror game against a human player. We developed two strategies. The former, based on adaptive control, was shown to be effective to achieve temporal correspondence between the motion of the virtual player and that of the human individual. Convergence of the algorithm was proved. However, we noted that such strategy does not allow the VP to exhibit some desired kinematic features (individual motor signature) of a given human player. To overcome this limitation, we proposed a different strategy based on the iterative solution of an appropriate optimal control problem. After proving boundedness and convergence of this additional approach, we tested its effectiveness experimentally. We showed that the proposed strategy is able to drive the VP so as to play the game both as leader or follower while matching well the individual motor signature of a given individual. Furthermore, we carried out a comparison with other existing models, confirming the effectiveness of the proposed approach. We wish to emphasize that our approach opens the possibility of making VPs, each modeling a different individual, play against each other and produce in silico experiments. This can reduce the cost and time of carrying out joint action experiments and can be effectively used to test different human-machine interaction scenarios via the mirror game.

The algorithm implementation for a real robot platform is the subject of ongoing research, which inevitably requires the technical consideration of robot actuators for stochastic disturbances [38], unknown actuator dynamics [39] and vibration suppression [40]. Finally, we are also exploring the possibility of combining adaptive and optimal control strategies, thus allowing the parameters of the optimal control to vary over time depending on the interaction with the other agent.

\section{ACKNOWLEDGMENT}

This work was funded by the European Project AlterEgo FP7 ICT 2.9 - Cognitive Sciences and Robotics, Grant Number 600610. The authors wish to thank Prof. Benoit Bardy, Prof. Ludovic Marin and Dr. Robin Salesse at EuroMov, University of Montpellier, France for all the insightful discussions and for collecting some of the experimental data that is used to validate the approach presented in this paper.

\section{REFERENCES}

[1] K. Johnstone, Impro: Improvisation and the theatre. Routledge, 2012

[2] L. Noy, E. Dekel, and U. Alon, "The mirror game as a paradigm for studying the dynamics of two people improvising motion together," Proceedings of the National Academy of Sciences, vol. 108, no. 52, pp. 20947-20952, 2011.

[3] V. S. Folkes, "Forming relationships and the matching hypothesis," Personality and Social Psychology Bulletin, vol. 8, no. 4, pp. 631-636, 1982.

[4] D. Lakens and M. Stel, "If they move in sync, they must feel in sync: Movement synchrony leads to attributions of rapport and entitativity," Social Cognition, vol. 29, no. 1, p. 1, 2011.

[5] S. S. Wiltermuth and C. Heath, "Synchrony and cooperation," Psychological Science, vol. 20, no. 1, pp. 1-5, 2009.

[6] S. Hussain, S. Q. Xie, and P. K. Jamwal, "Adaptive impedance control of a robotic orthosis for gait rehabilitation," IEEE Transactions on Cybernetics, vol. 43, no. 3, pp. 1025-1034, 2013.
[7] G. Dumas, G. C. de Guzman, E. Tognoli, and J. S. Kelso, "The human dynamic clamp as a paradigm for social interaction," Proceedings of the National Academy of Sciences, vol. 111, no. 35, pp. E3726-E3734, 2014.

[8] C. Zhai, F. Alderisio, K. Tsaneva-Atanasova, and M. di Bernardo, "A model predictive approach to control the motion of a virtual player in the mirror game," in 2015 54th IEEE Conference on Decision and Control (CDC). IEEE, 2015, pp. 3175-3180.

[9] P. E. W. Ouwehand and C. L. E. Peper, "Does interpersonal movement synchronization differ from synchronization with a moving object?" Neuroscience letters, vol. 606, pp. 177-181, 2015.

[10] Z. Liu, C. Chen, Y. Zhang, and C. P. Chen, "Adaptive neural control for dual-arm coordination of humanoid robot with unknown nonlinearities in output mechanism," IEEE Transactions on Cybernetics, vol. 45, no. 3 , pp. 507-518, 2015.

[11] M. I. Jordan and D. M. Wolpert, "Computational motor control," 1999.

[12] F. M. Drop, D. M. Pool, H. J. Damveld, M. M. van Paassen, and M. Mulder, "Identification of the feedforward component in manual control with predictable target signals," IEEE Transactions on Cybernetics, vol. 43, no. 6, pp. 1936-1949, 2013.

[13] V. A. Laurense, D. M. Pool, H. J. Damveld, M. R. M. van Paassen, and M. Mulder, "Effects of controlled element dynamics on human feedforward behavior in ramp-tracking tasks," IEEE Transactions on Cybernetics, vol. 45, no. 2, pp. 253-265, 2015.

[14] M. Desmurget and S. Grafton, "Forward modeling allows feedback control for fast reaching movements," Trends in Cognitive Sciences, vol. 4, no. 11, pp. 423-431, 2000.

[15] P. Słowiński, E. Rooke, M. di Bernardo, and K. Tanaseva-Atanasova, "Kinematic characteristics of motion in the mirror game," in 2014 IEEE International Conference on Systems, Man, and Cybernetics (SMC). IEEE, 2014, pp. 748-753.

[16] P. Słowiński, C. Zhai, F. Alderisio, R. Salesse, M. Gueugnon, L. Marin, B. G. Bardy, M. di Bernardo, and K. Tsaneva-Atanasova, "Dynamic similarity promotes interpersonal coordination in joint action," Journal of The Royal Society Interface, vol. 13, no. 116, p. 20151093, 2016.

[17] Z. Zhang, A. Beck, and N. Magnenat-Thalmann, "Human-like behavior generation based on head-arms model for robot tracking external targets and body parts," IEEE Transactions on Cybernetics, vol. 45, no. 8, pp. 1390-1400, 2015.

[18] J. S. Kelso, G. C. de Guzman, C. Reveley, and E. Tognoli, "Virtual partner interaction (vpi): exploring novel behaviors via coordination dynamics," PloS one, vol. 4, no. 6, p. e5749, 2009.

[19] J. Hölldampf, A. Peer, and M. Buss, "Virtual partner for a haptic interaction task," in Human Centered Robot Systems. Springer, 2009, pp. 183-191.

[20] H. Haken, J. S. Kelso, and H. Bunz, "A theoretical model of phase transitions in human hand movements," Biological Cybernetics, vol. 51 , no. 5 , pp. 347-356, 1985.

[21] B. Kay, J. Kelso, E. Saltzman, and G. Schöner, "Space-time behavior of single and bimanual rhythmical movements: Data and limit cycle model." Journal of Experimental Psychology: Human Perception and Performance, vol. 13, no. 2, p. 178, 1987.

[22] A. Fuchs, V. K. Jirsa, H. Haken, and J. S. Kelso, "Extending the hkb model of coordinated movement to oscillators with different eigenfrequencies," Biological Cybernetics, vol. 74, no. 1, pp. 21-30, 1996

[23] R. C. Schmidt and M. J. Richardson, "Dynamics of interpersonal coordination," in Coordination: Neural, behavioral and social dynamics. Springer, 2008, pp. 281-308.

[24] C. Zhai, F. Alderisio, P. Słowiński, K. Tsaneva-Atanasova, and M. di Bernardo, "Design of a virtual player for joint improvisation with humans in the mirror game," PloS one, vol. 11, no. 4, p. e0154361, 2016.

[25] F. Alderisio, B. G. Bardy, and M. di Bernardo, "Entrainment and synchronization in networks of rayleigh-van der pol oscillators with diffusive and haken-kelso-bunz couplings," Biological Cybernetics, vol. 110, no. 2, pp. 151-169, 2016.

[26] F. Alderisio, D. Antonacci, C. Zhai, and M. di Bernardo, "Comparing different control approaches to implement a human-like virtual player in the mirror game," in 15th European Control Conference (ECC), 2016, pp. 216-221.

[27] E. Levina and P. Bickel, "The earth mover's distance is the mallows distance: some insights from statistics," in Computer Vision, 2001. ICCV 2001. Proceedings. Eighth IEEE International Conference on, vol. 2. IEEE, 2001, pp. 251-256.

[28] C. K. Ahn, S.-T. Jung, S.-K. Kang, and S.-C. Joo, "Adaptive $\mathscr{H}_{\infty}$ synchronization for uncertain chaotic systems with external distur- 
bance," Communications in Nonlinear Science and Numerical Simulation, vol. 15, no. 8, pp. 2168-2177, 2010.

[29] C. Zhai, F. Alderisio, K. Tsaneva-Atanasova, and M. di Bernardo, "A novel cognitive architecture for a human-like virtual player in the mirror game," in 2014 IEEE International Conference on Systems, Man, and Cybernetics (SMC). IEEE, 2014, pp. 754-759.

[30] J. M. Pak, C. K. Ahn, Y. S. Shmaliy, P. Shi, and M. T. Lim, "Switching extensible fir filter bank for adaptive horizon state estimation with application," IEEE Transactions on Control Systems Technology, vol. 24 no. 3, pp. 1052-1058, 2016.

[31] Q. Zhou, H. Li, C. Wu, L. Wang, and C. K. Ahn, "Adaptive fuzzy control of nonlinear systems with unmodeled dynamics and input saturation using small-gain approach," IEEE Transactions on Systems, Man, and Cybernetics: Systems, 2016.

[32] J. Diedrichsen, R. Shadmehr, and R. B. Ivry, "The coordination of movement: optimal feedback control and beyond," Trends in Cognitive Sciences, vol. 14, no. 1, pp. 31-39, 2010.

[33] D. S. Naidu, Optimal control systems. CRC press, 2002.

[34] J. Guna, G. Jakus, M. Pogačnik, S. Tomažič, and J. Sodnik, "An analysis of the precision and reliability of the leap motion sensor and its suitability for static and dynamic tracking," Sensors, vol. 14, no. 2, pp. 3702-3720, 2014.

[35] T. Kreuz, F. Mormann, R. G. Andrzejak, A. Kraskov, K. Lehnertz, and P. Grassberger, "Measuring synchronization in coupled model systems: A comparison of different approaches," Physica D: Nonlinear Phenomena, vol. 225, no. 1, pp. 29-42, 2007.

[36] S. J. Orfanidis, Optimum signal processing: an introduction. Macmillan publishing company, 1988.

[37] B. Kralemann, L. Cimponeriu, M. Rosenblum, A. Pikovsky, and R. Mrowka, "Phase dynamics of coupled oscillators reconstructed from data," Physical Review E, vol. 77, no. 6, p. 066205, 2008.

[38] C. Chen, Y. Zhang, C. P. Chen, S. Xie et al., "Asymptotic fuzzy tracking control for a class of stochastic strict-feedback systems," IEEE Transactions on Fuzzy Systems, 2016.

[39] C. Chen, Z. Liu, Y. Zhang, C. P. Chen, and S. Xie, "Saturated nussbaum function based approach for robotic systems with unknown actuator dynamics," IEEE Transactions on Cybernetics, 2015.

[40] C. Chen, Z. Liu, Y. Zhang, and C. L. P. Chen, "Modeling and adaptive compensation of unknown multiple frequency vibrations for the stabilization and control of an active isolation system," IEEE Transactions on Control Systems Technology, vol. 24, no. 3, pp. 900-911, 2016.

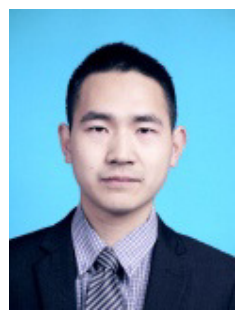

Chao Zhai received his Bachelor degree in Automation Engineering from Henan University in 2007 and earned his Master degree in Control Theory and Engineering from Huazhong University of Science and Technology in 2009. He received his Ph.D. in Complex System and Control from the Institute of Systems Science, Chinese Academy of Sciences in 2013. Between July 2013 and August 2015 he was a Post-Doctoral Fellow at the University of Bristol, UK. Currently, he is working as a Research Fellow at Singapore-ETH Centre. His research revolves around motor coordination in biological systems, cooperative control, cascading failure in power systems and evolutionary game theory.

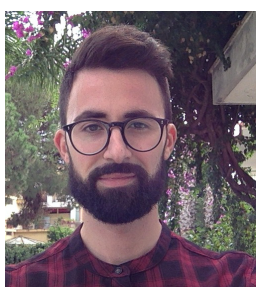

Francesco Alderisio received his M.Sc. degree in Control Engineering in December 2013 by the University of Naples Federico II, Italy. Between January 2014 and July 2016 he was involved in the European Project AlterEgo, and in October 2014 he started his $\mathrm{Ph} . \mathrm{D}$. in Engineering Mathematics at the University of Bristol, UK. His current research interests include human-robot interaction, as well as analysis and control of synchronization in complex networks with applications to human movement coordination.

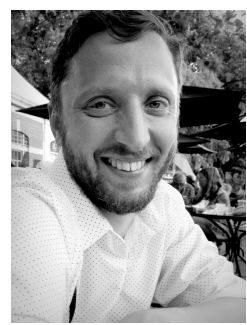

Piotr Słowiński received his M.Sc. in Biomedical Engineering from the University of Warsaw in 2006. $\mathrm{He}$ received his Ph.D. in Engineering Mathematics from the University of Bristol in 2011. Currently, he is working as a Research Fellow at the University of Exeter. His research interests include mathematics for healthcare, mathematical neuroscience, dynamical systems theory and complex systems.

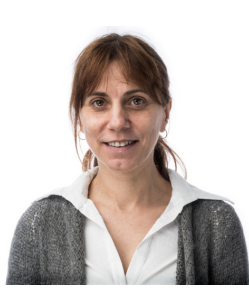

Krasimira Tsaneva-Atanasova received her $\mathrm{Ph}$.D form the University of Auckland, New Zealand, in October 2004, and subsequently held Postdoctoral Research Fellowships at the National Institutes of Health in Bethesda, MD, USA, from November 2004 to April 2006, and at Ecole Normale Superieure in Paris, France, from May 2006 to July 2007. She was appointed as a lecturer in August 2007 and promoted to a Reader in Applied Mathematics in August 2012 in the Department of Engineering Mathematics at the University of Bristol, before moving to the University of Exeter in July 2013. She is currently a professor of Mathematics for Healthcare at the Department of Mathematics at Exeter. She uses mathematical models to study the mechanisms of cell signalling and communication. She is also engaged in collaborative projects in the area of movement science and experimental psychology.

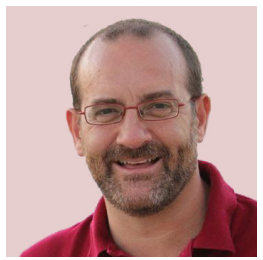

Mario di Bernardo (SMIEEE '06, FIEEE 2012) is currently Full Professor of Automatic Control at the University of Naples Federico II, Italy. He is also Professor of Nonlinear Systems and Control at the University of Bristol, UK and Honorary Professor of Control at Fudan University, China. In 2007 he was bestowed the title of "Cavaliere" of the Order of Merit of the Italian Republic for scientific merits from the President of Italy. In 2012 he was elevated to the grade of Fellow of the IEEE for his contributions to the analysis, control and applications of nonlinear systems and complex networks. In 2009, he was elected President of the Italian Society for Chaos and Complexity for the term 2010-2013. He was re-elected in 2010 for the term 2014-2017. In 2006 and again in 2009 he was elected to the Board of Governors of the IEEE Circuits and Systems Society. From 2011 to 2014 he was Vice President for Financial Activities of the IEEE Circuits and Systems Society. In 2015 he served as appointed member of the Board of Governors of the IEEE Control Systems Society. His research interests include the analysis, synchronization and control of complex network systems; the analysis and control of hybrid and piecewisesmooth dynamical systems; nonlinear dynamics, nonlinear control theory and applications to engineering and synthetic biology. He received funding from several institutions including the EU, the Italian Ministry of University and Research, the UK Research Councils and Industry for a total amount of over $8 \mathrm{M}$ Euros. 